\title{
QUANTITATION OF THE HUMAN BASAL GANGLIA : WITH POSITRON EMISSION TOMOGRAPHY
}

\author{
B. Bendriem, SL Dewey, DJ Schlyer, AP Wolf and ND Volkow* \\ Dept of Chemistry and *Medical Dept, BNL, UPTON, NY 11973.
}

\section{Abstract}

The accurate measurement of the concentration of a radioisotope in small structures with PET requires a correction for quantitation loss due to the partial volume effect and the effect of scattered radiation. To evaluate errors associated with measures in the human basal ganglia (BG) we have built a unilateral model of the BG that we have inserted in a $20 \mathrm{~cm}$ cylinder. The recovery coefficient $(R C=$ measured activity/true activity) for our BG phantom has been measured on a CTI tomograph (model 931-08/12) with different background concentrations (contrast) and at different axial locations in the gantry. The BG was visualized on 4 or 5 slices depending on its position in the gantry and on the contrast used. The RC was 0.75 with no background (contrast equal to 1.0). Increasing the relative radioactivity concentration in the background increased the $R C$ from 0.75 to 2.00 when the contrast was -0.7 (BG < Background). The RC was also affected by the size and the shape of the region of interest (ROI) used (RC from 0.75 to 0.67 with ROI size from 0.12 to $1.41 \mathrm{~cm}^{2}$ ). These results show that accurate RC correction depends not only on the volume of the structure but also on its contrast with its surroundings as well as on the selection of the ROI. They also demonstrate that the higher the contrast the more sensitive to axial positioning PET measurements in the BG are. These data provide us with some information about the variability of PET measurements in small structure like the BG and we have proposed some strategies to improve the reproducibility.

\section{INTRODUCTION}

A Positron Tomograph (PT) is an elaborated nuclear counting device that measures the concentration of a positronemitter in a volume element of a given object. A major effort has been devoted to the development of quantitative Positron Emission Tomography (PET) instrumentation to provide accurate data from which functionality can be derived [1]. Indeed from the distribution in space and time of a radiolabelled molecule, biochemical functions and physiological parameters can be assessed through the use of blood chemistry and biomathematical models that describe kinetics processes undergone by the tracer in the organ studied.

This research was carried out at Brookhaven National Laboratory under contract DE-AC02-76CH00016 with the U.S. Department of Energy and supported by its office of Health and Environmental Research.

B. Bendriem new address as of 11/01/90 is Service Hospitalier Frédéric Joliot, C.E.A., hopital d'Orsay, 91406 ORSAY FRANCE.
The study of receptor-ligand interaction and the measurement of receptor densities and affinity constants in vive has been investigated for more than 10 years [2], [3], [4]. The knowledge of these parameters is of prime importance for the understanding of fundamental biochemistry and could constitute a valuable tool for the clinical characterization of pathological states. However the precision of the inethod used to derive them must become reliable enough to prevent findings of contradictory results [5]. Such precision will be achieved through the combined effort of chemists, biochemists, biomathematicians and physicist if they can label and use the pertinent radiotracer [6], design and develop the optimal protocols and adequate models [7], improve the instrumentation and implement the necessary correction schemes to improve image quantitation.

Errors in quantitation are accentuated when measuring activity concentration in small structures such as the human basal ganglia (BG). It is known that structures whose dimensions are smaller than two times the resolution (full width half $\max$ (FWHM)) of the instrument in all directions cannot be exactly quantitated [1]. The notion of recovery coefficients (RC) for a given object has been introduced as the ratio of the measured over the true activity concentration in the object [8]. The RC of various structures in the brain have been previousiy evaluated as a function of resolution of PET instrument assuming that the structure is located at the center of the tomographic slice, and that its shape can be approximated by a simple geometric form [9]. The effect of contrast gradients in the surrounding tissues on RC has also been emphasized [10]. The contribution of all these factors needs to be evaluated in order to avoid misinterpretation of PET data. Indeed in a kinetic study involving a tracer that specifically binds to receptors in the BG the image contrast increases with time and the errors in the PET measurements are not systematic.

A large number of tracers have been developed for probing different aspects of the dopaminergic system [11]. For example, tracers for probing dopamine metabolism, dopamine reuptake and dopamine receptors are all under active investigation. The primary region of interest in the brain, for the dopaminergic system, is the basal ganglia, a subcortical structure of irregular shape having a total volume of about 13 $\mathrm{cm}^{3}$ [12], which contains the highest concentration of postsynaptic dopamine D2 receptors. Therefore an assessement of the errors incurred in measuring the concentration of radioactivity in the BG and an understanding of the quantitative limitations in these measurements is of major importance. In order to address this issue from the perspective of the accuracy and the precision of the measurements, we have built a unilateral model of that brain

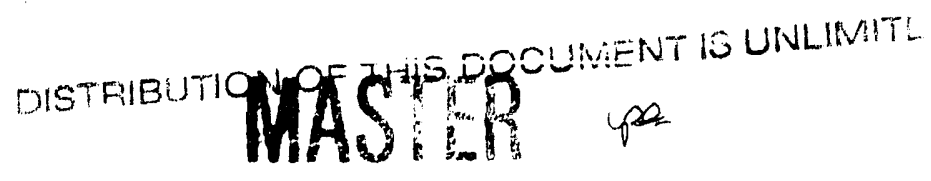


structure. We have conducted a series of PET experiments on a CTI tomograph (model 931/08-12) to evaluate, at various contrast levels, the effect on quantitation of the limited resolution of PET, of scattered radiation and of slight mispositioning of the patient in the axial direction of the gantry. We had previously shown that as axial resolution of PET camera increases small mispositions (on the order of 2 $\mathrm{mm}$ ) can introduce large errors in the quantitation of small objects whose shape is irregular within the tomographic slice [13].

Based on our initial results, we were able to assess the variability of our measurement and to identify some sources of these variations. We propose here some techniques to reduce their influence.

\section{MATERIALS AND METHODS.}

\section{A. The basal ganglia model}

A unilateral basal ganglia (BG) model was built by using horizontal sections of a brain atlas (The Structure of the Human Brain. a Photographic Allas by DeAmond, Fusco and Dewey) as a reference for anatomy. Briefly, modelling clay (5$7 \mathrm{~mm}$ thick) was placed directly on the photographic images such that the entire structure, including the head of the caudate nucleus, putamen and globus pallidus was completely covered on every image upon which they appeared. Subsequently these clay molds were stacked together from the most caudal level to the most rostral end of the basal ganglia. The edges were smoothed carefully and the whole clay mold was cast in rubber where it stood for 24 hours. Using paraffin, another mold of the basal ganglia was made from the rubtor cast. The latter mold was then used for the final step of the process during which it was covered with li id acrylic. Upon completion of the outer surface a hole was drilled and the paraffin was melted and thoroughly removed from the inner cavity. The hole was subsequently used for the insertion of radioactive solution. Our BG model had an internal volume of $6.5 \mathrm{~cm}^{3}$ consistent with the average volume of a unilateral human bas. [12] and could be rigidly attached inside a $20 \mathrm{~cm}$ cylinder. As with images of the distribution of $\mathrm{N}$-Methylspiroperidol (NMSP, a dopamine D2 receptor antagonist) in the human brain [14], the BG phantom appeared, on the CTI 93I, on 4 or 5 slices depending on the contrast and on its axial position in the field of view.

\section{B. The tomograph}

The experiments were conducted on: a CTI tomiograph (model 931-08/12) which has been described elsewhere [15]. Briefly it is an eight ring system capable of producing 15 slices simultaneously. It is a whole sody unit and each ring has a diameter of $102 \mathrm{~cm}$. There are 51 ?. Bismuth Germanate (BGO) crystals per ring and then 4096 total crystals which are assembled in blocks of 8 (in-plane) by 4 (axial) crystals coupled with 4 photomultiplier tubes. The transverse resolution of the system, at the center of the gani's' is 6.0 $\mathrm{mm}$ (full width half-max (FWHM), obtained from the image of a line source reconstructed with a Ramp filter). 'n our standard operations, however, we use a Hanning filter (with a frequency cutoff of .5 cycle/pixel) to smooth the projection data and our practical transverse resolution is $8.5 \mathrm{~mm}$. The axial resolution is $6.2 \mathrm{~mm}$ (FWHM) for cross slices, $6.7 \mathrm{~mm}$ for direct slices. The separation between slices is $6.75 \mathrm{~mm}$ and the total axial length scanned is $108 \mathrm{~mm}$. The image pixel size is adjustable and was set to $1.565 \mathrm{~mm}$ while the matrix size was $128 x$ !28.

\section{The experimental procedure}

The scanning was performed as done for clinical procedures. Attenuation correction was computed with measured correction factors. A transmission scan of the phantom was acquired, using 8 thin annular sources filled with solid germanium-68 (half-life $=275$ days). The average number of true counts (including scatter counts) for the reference as well as the transmission scan was $5.10^{7}$ per slice. We did not correct the transmission data for scattered radiation. However, from transmission measurements of cylindrical phantoms $(13 \mathrm{~cm}$ and $20 \mathrm{~cm}$ ) filled with water we have evaluated a linear coefficient of attenuation of water (for $0.511 \mathrm{MeV}$ gammarays) of $0.0947 \pm 0.0013 \mathrm{~cm}^{-1}(2 \%$ lower than the true value of $0.0967 \mathrm{~cm}^{-1}$ ).

Ten measurements with a single isotcpe were made using Fluorine-18 (F-18; half-life $=109.8 \mathrm{~min}$.) in the BG. For the first five experiments, there was no activity in the background (contrast equal to 1) and there was $2.69 \mathrm{uci} / \mathrm{ml}(0.1 \mathrm{MBq} / \mathrm{ml})$ in the BG. The total activity in the field of view was about 17.5 uci and, at this low level of radioactivity, the dead time losse's were less than $1 \%$ and no random were collected. Two scans were taken with the phantom at the same position in the gantry while the other three were taken shifting the position of the phantom (by moving the computer controlled bed) along the axial direction by steps of $2 \mathrm{~mm}$. The same procedure was followed for the other five measurements except that they were done with activity in the background (BK). The ratio $\mathrm{BG} / \mathrm{BK}$ was equal ir 3.5 and the true contrast, defined here as $\mathrm{CR}=(3 \mathrm{G}-\mathrm{BK}) /(\mathrm{BG}+\mathrm{BK})$, was equal to 0.55 . The activity in the background was $0.36 \mathrm{uci} / \mathrm{ml}(13.3 \mathrm{MBq} / \mathrm{ml})$ which corresponded to about $1 \mathrm{mci}$ in the field of view. The dead time losses were in the range $10-12 \%$ and the randoms were about $34 \%$ of the true plus scatter counts. The ten scans were five minutes long. The true activity concentrations in the $B G$ and in the BK were measured in a well counter cross calibrated with the tomograph and whose precision was $\pm 2 \%$. All the images were corrected on line for dead time losses and random registration, and sinograms were processed to compensate for the attenuation of the $0.511 \mathrm{MeV}$ photons as described above and for the variations in sensitivity of individual detectors. They were also corrected for decay to a fix reference time.

In another experiment the dual isotope procedure proposed by Cooke and Evans [16] was used. Carbon-11 (C-11; halflife $=20.4 \mathrm{~min}$ ) was used in the BG and F-18 in the BK. The phantom was located at its original position and the initial contrast measured on the well counter was 0.90 $(B G / B K=19.5)$. The initial activity concentration in the $B G$ 
was $32.7 \mathrm{uci} / \mathrm{ml}(1.2 \mathrm{MBq} / \mathrm{ml})$ while that of the BK was 1.68 $\mathrm{uci} / \mathrm{ml}(0.062 \mathrm{MBq} / \mathrm{ml})$. Initially there was about $5 \mathrm{mci}$ in the field of view of our tomograph, the dead time losses were about $60 \%$ and the randoms were $18 \%$ of the true plus scatter counts. Twenty nine scans (6.8 minutes each) were acquired in a dynamic mode and the contrast at the end of the experiment was $-0.83(\mathrm{BG} / \mathrm{BK}=0.09)$. The images were corrected for random dead time loss and attenuation as described above but not for radioactive decay.

\section{Region of Interest definition}

Two ROIs were defined for the BG on the tomographic slice where it appeared with the apparent highest concentration. The first one was circular (CRON), contained 5 pixels and was located in the structure in such a way that edge effects were minimized. The second one was irregular and followed the contour of the BG as it appeared on the PET image. It contained at least 75 pixels and was designated as the aratomical ROI (AROI). It should be noted that this type of definition is subjective and depends largely on the investigator as well as on the color scale used to display the image. We have used thi. approach to compare the results between a large and a small ROI and to provide some insight on the importance of the strategy used for the definition of the ROI.

A large Region of Interest (ROI) of 1200 pixels was drawn in the background (BROI) on the slice where the BG was defined. Spill over effects from the BG was minimized by allowing at least 3 times the resolution (FWHM) between the region and the $B G$. Values obtained for $B K$ were used to assess the precision of the correction for random, dead time and attenuation on our scanner and to insure the proper cross calibration with the well counter.

\section{RESULTS AND DISCUSSION}

\section{A. The single isotope experiments}

In all our subsequent tables we have reported $\mathrm{RC}$ values defined as the ratio of measured value on the PET image over the measured value on the well counter. It should be pointed out that the concentration of radioisotope in the BG was assayed once at the beginning of the ten single isotope experiments, and that the same "true concentration " value was used for all our measurements.

In table I we have reported the results obtained for the first five static experiments when there was no activity in the background. RC values vary from 0.65 to 0.80 (23\% variation). Although our model has a larger volume than that of a cell with dimensions equal to 2 times the resolution (in the 3 directions) of the tomograph $\left(3.8 \mathrm{~cm}^{3}=1.7 \times 1.7 \times 1.3\right)$, there is a quantitation loss which is larger than 20\%. Part of it is due to the effect of scattered radiation and part of it to the partial volume effect since the shape of our model is not uniform within the tomographic slice. As can be seen in the first column of table I the values obtained with the small circular ROI are sensitive to a small shift of $2 \mathrm{~mm}$ in the axial direction of the gantry. The average CROI value is $0.75 \pm$ 0.03 (mean \pm SD), which corresponds to a $25 \%$ quantitation loss for the small circular ROI. The reproducibility of the RC value at position 0 strongly suggests that the variations are due to the effect of axial positioning rather than to the statistical fluctuations. They are less pronounced but still noticeable for AROI where the average is $0.67 \pm 0.02$ (mean \pm SD). The quantitation loss is this time $33 \%$ and our data illustrate the magnitude of variations that can be introduced by the type of ROI.

We have applied a scatter correction algorithm on our data. which consisted of a simple deconvolution assuming an invariant exponential shape for the scattered radiation distribution [17]. The parameters of the exponential were determined from the measurement of a Ge-68 line source centered in the $20 \mathrm{~cm}$ cylinder. The scatter fraction was 0.10 and the exponential slope was $0.13 \mathrm{~cm}^{-1}$. The correction resulted in a consistent $9 \%$ increase of all the $R C$ values.

Table I

$\mathrm{RC}$ vajues for the $\mathrm{BG}$ as a function of bed position and type of ROI (BK $=0$, bed positions are $2 \mathrm{~mm}$ apart)

\begin{tabular}{|c|c|c|}
\hline $\begin{array}{c}\text { BED } \\
\text { POSITION }\end{array}$ & $\begin{array}{c}\text { CROI } \\
\text { (5 Pixels) }\end{array}$ & $\begin{array}{c}\text { AROI } \\
(>75 \text { Pixels })\end{array}$ \\
\hline 0 & 0.74 & 0.68 \\
\hline 1 & 0.71 & 0.65 \\
\hline 2 & 0.76 & 0.66 \\
\hline 3 & 0.80 & 0.70 \\
\hline 0 & 0.74 & 0.69 \\
\hline
\end{tabular}

In table II we have reported the RC values for $\mathrm{BG}$ and $\mathrm{BK}$ when the true contrast was 0.55 . From the values in the last column we can evaluate that, at the level of radioactivity used, the precision of our PET measurements (which includes the effectiveness of the various correction applied to the raw data) is $2 \%$.

Values for CROI and AROI are larger and less sensitive to axial shift than the values reported in table I. This observation was somehow expected since radioactive sources, located at different axial position within the tomographic slice, and originating from both the $\mathrm{BG}$ and the $\mathrm{BK}$ are integrated in the PET measurements. The partial volume effect variations due to axial shift are then minimized by the presence of activity in the background. The quantitation loss is $21 \%$ for the circular ROI $(0.79 \pm 0.02$; mean $\pm S D)$ and $28 \%$ for the anatomical $R O I$ $(0.72 \pm 0.01$; mean $\pm S D)$. The increase of the $R C$ is a result of the influence of the background activity on the $B G$ measurement. The variations of the $B G$ measurement, when using a large ROI, are similar to that of the background. They are slightly better than for a small $\mathrm{ROI}$ at the expense of a greater quantitation loss. The results show that at a true 
contrast value of 0.55 , the influence of small axial shift is masked by the influence of the background activity.

\section{Table II}

$R C$ values for the $B G$ and the bri as a function of bed position and type of ROI (BG/BK=3.5, bed positions are $2 \mathrm{~mm}$ apart)

\begin{tabular}{|c|c|c|c|}
\hline $\begin{array}{c}\text { BED } \\
\text { POSITION }\end{array}$ & $\begin{array}{c}\text { CROI } \\
\text { (5 pixels) }\end{array}$ & $\begin{array}{c}\text { AROI } \\
(>75 \text { pixels })\end{array}$ & $\begin{array}{c}\text { BROI } \\
(1200 \text { pixels })\end{array}$ \\
\hline 0 & 0.79 & 0.72 & 1.00 \\
\hline 1 & 0.80 & 0.72 & 0.99 \\
\hline 2 & 0.79 & 0.73 & 1.02 \\
\hline 3 & 0.82 & 0.72 & 1.02 \\
\hline 0 & 0.77 & 0.70 & 0.98 \\
\hline
\end{tabular}

Besides the constant quantitation loss that affects the BG measurement which is mainly due its size and shape our data outline that variations in the $10 \%$ range of the values obtained from a single experiment can be explained by technical factors such as the size of ROI, the image contrast or the subject positioning. These factors will affect measurements in small regions like the $B G$ and should be considered when comparing different populations or when testing individual several times under different activation states. We did not include in our analysis the effects of non uniform resolution in a circular ring of detectors [18] since these effects are minimal, when a system with a large diameter ring of detector is used for brain scanning, provided the patient is well centered in the field of view of the tomograph. The following section further elaborates on the importance of the contrast on the determination of $\mathrm{RC}$.

\section{B. The dual isotope experiment}

The results obtained from the dual isotope experiment are detailed in figures 1 and 2 . Figure 1 plots the time activity curve obtained from CROI and BROI along with the calculated curve obtained from the initial concentration measured in the well counter and from the known half-life of each isotope. The measured hackground agrees nearly exactly with the calculated one and the RC for BK fluctuates between 0.97 and 1.02 which again is an indication of the precision of the various corrections applied on the raw data. The BG however cannot be fitued with a monoexponential and the $\mathrm{RC}$ of the $\mathrm{BG}$ varies from 0.75 to (when $B G \gg B K$ ) to more than 2.0 (when $B G \ll B K$ ). The initial value of $R C$ is consistent with the results obtained from the single isotope experiments.

Figure 2 represents the variations of the $\mathrm{RC}$ of the $\mathrm{BG}$ as a function of the true contrast in the image and demonstrates the importance of the radioactivity in the surroundings. Due to the effect of scattered radiation RC factors greater than one are observed when the activity concentration in the BG is lower than that in the background (negative contrast). The noise on this part of the curve increases as the activity in the $\mathrm{BG}$ decreases. Such situations however are unlikely in clinical studies except in some pathological states (such as stroke for example).

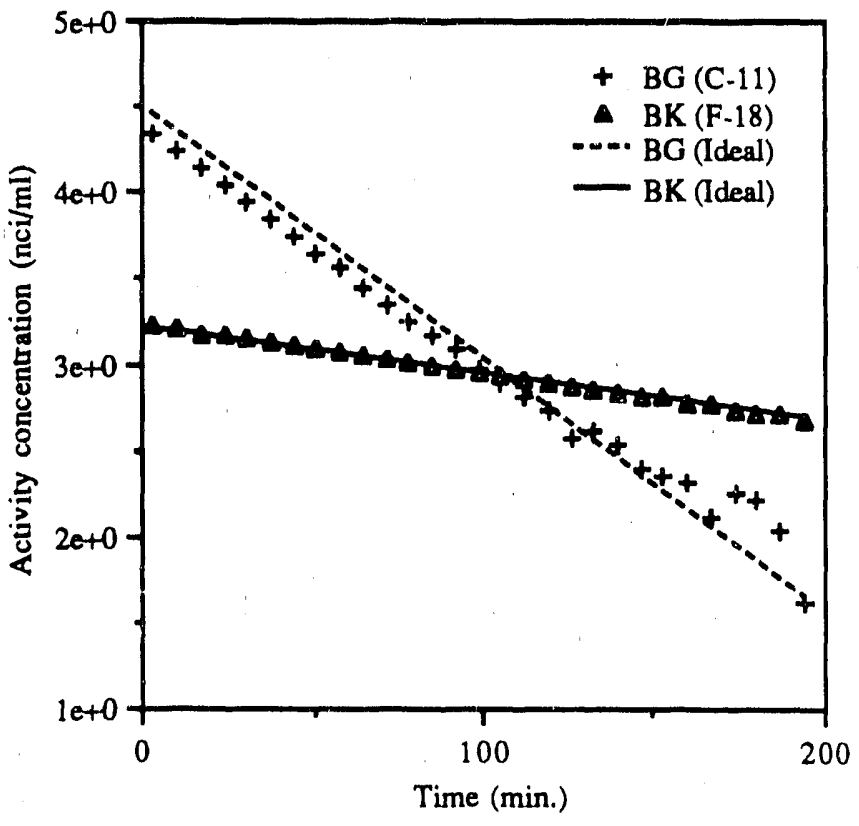

Fig. 1 Comparison between predicted and measured time activity curve (dual isotope experiment)

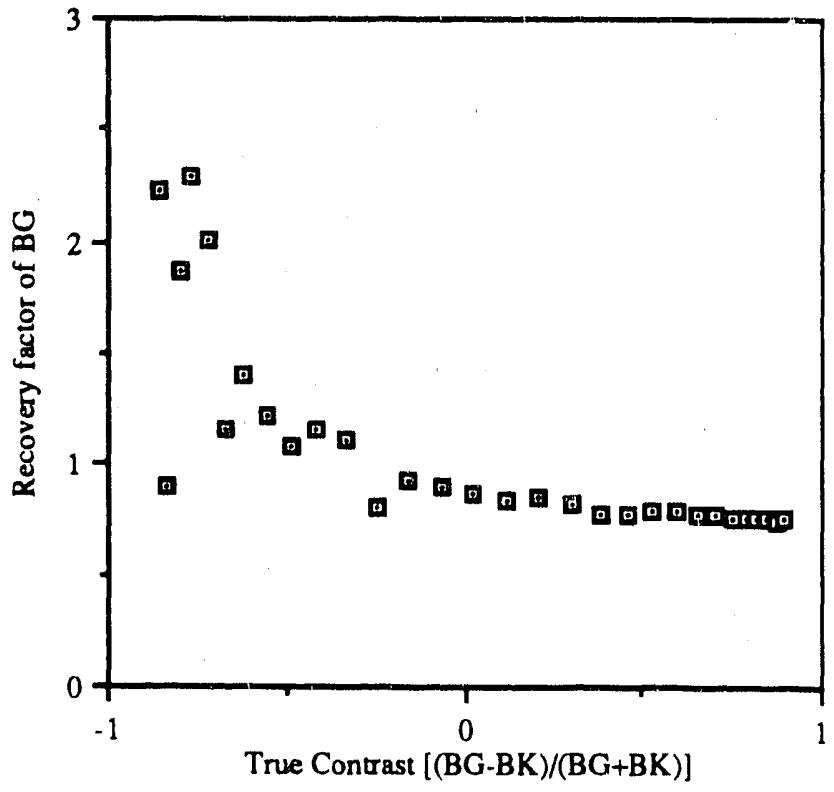

Fig. 2 Variation of the RC of the BG as a function of the true contrast in the phantom

Our data also show that a constant correction factor cannot be applied and the consequences of this result on the evaluation of physiological parameters should be further 
investigated. Indeed on the images of the distribution in time of [F-18]-NMSP in the basal ganglia, the contrast usually varies from 0.0 (just after injection) to 0.7 ( 4 hours after injection) which corresponds to a variation in RC from 0.90 to 0.75 .

In order to describe accurately the variations of BK and BG with time we have tried to fit our data to the following equations :

$B K=B K o \cdot \exp (-t \cdot \ln (2) / 109.8)$

$B G=a \cdot B K o \cdot \exp (-t . \ln (2) / 109.8)+b . B G o \cdot \exp (-t \cdot \ln (2) / 20.4)(2)$

where $t$ is the mid-scan time in minutes, BKo and $B C^{\prime} 0$ are the initial activity concentrations. Strictly speaking, using the mid-scan time is incorrect since the radioactive decay follows an exponential decrease. However since our scans were 6.8 minutes long, the error introduced is $0.2 \%$ for the C-11 compartment. The parameter a describes the influence of the background on the measurement of the BG v/hile the the parameter $b$ describes the quantitation loss in the BG. Ideally a should be equal to 0 and $b$ to 1 . We have reconstructed our dynamic data with three different algorithms. Table III reports the values of $a$ and $b$ (determined by non linear regression) from the fit of each time activity curve generated. Using a Ramp filter, which improves the image transverse resolution from $8.5 \mathrm{~mm}$ to $6.0 \mathrm{~mm}$, did change the parameter $b$ from 0.75 to 0.77 but did not affect the parameter a. Application of a scatter correction however largely reduced the influence of the background activity and reduced the quantitation loss in the BG. These results suggest that the scattered radiation is an important component in the quantitation of the human basal ganglia and that that the effect of the limited resolution is mainly due its axial component.

\section{Table III.}

Variation of the bi-exponential parameters of the fit to the BG time activity curve as a function of the reconstruction mode.

\begin{tabular}{|c|c|c|}
\hline reconstruction mode & $\mathrm{a}$ & $\mathrm{b}$ \\
\hline $\begin{array}{c}\text { Hanning filter, } \\
\text { no scatter correction }\end{array}$ & 0.13 & 0.75 \\
\hline $\begin{array}{c}\text { Ramp Filter, } \\
\text { no scauter correction }\end{array}$ & 0.13 & 0.77 \\
\hline $\begin{array}{c}\text { Harning filter, } \\
\text { scatter correction }\end{array}$ & 0.05 & 0.81 \\
\hline
\end{tabular}

Figure 3 illustrates the agreement between the model fit and the measured data. Such an approach could be applied to the distribution of NMSP in the BG if the kinetic processes undergone in the adjacent tissues can be described by an homogeneous function. A constant fraction of this function could be removed from the BG time activity curve.

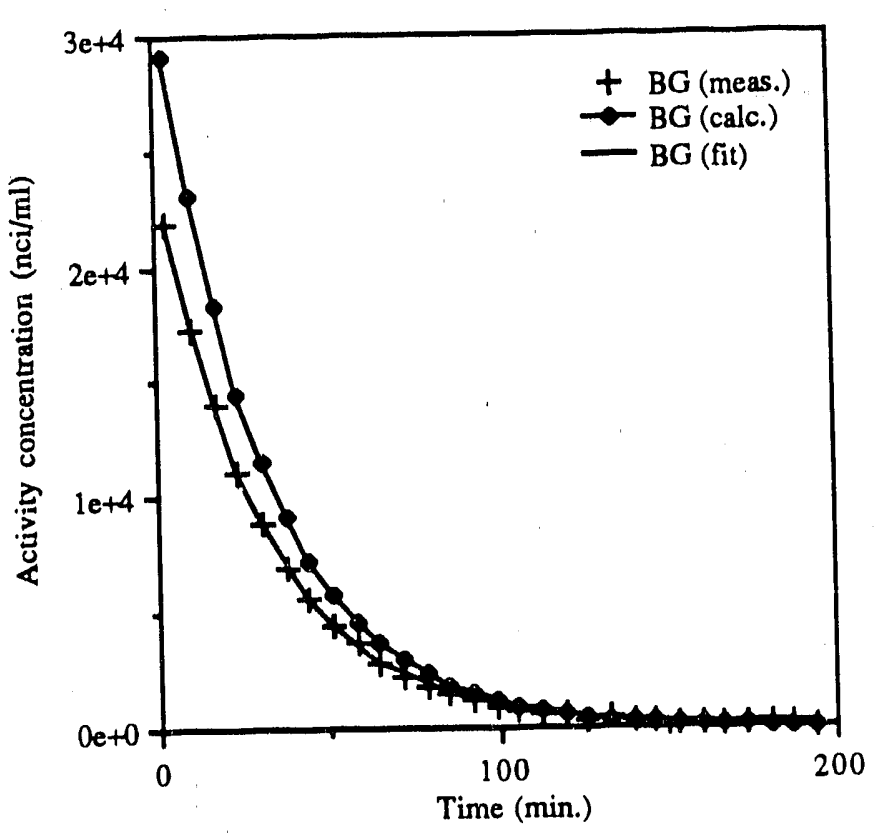

Fig. 3 Comparison between measured, calculated and fitted time activity curves in the BG

\section{REDUCTION OF THE DATA VARIATIONS}

As we have discussed it in the previous section variations in the measurement of the BG activity concentration are large in two situations : 1) when a small ROI is defined, 2) when the contrast with surrounding tissues is very high. Under these conditions, measured values are very sensitive to small changes in axial position $(2 \mathrm{~mm})$ which are very difficult to control in practice. This should be considered carefully especially in light of the fact that part of the basal ganglia (namely the head of caudate) is adjacent to the ventricles which are usually devoid of activity.

In order to minimize these distortions we have defined small circular regions of interest (5 pixels each) on all the tomographic slices where the BG appeared. Looking at the numerical values we have selected the slices with an appdrent concentration greater than $20 \%$ of the maximum value. In all five experiments when there was no background activity this corresponded systematically to the selection of three contiguous slices. We then averaged the three ROI values and called this new measure the integrated ROI value. Table IV compares the results obtained with the single ROI (as previously defined) versus the Integrated ROI. For all but one bed position the integrated ROI provides a stable value. The quantitation loss however is increased (from 25 to 43\%) since it reflects more the effect of limited resolution in the 3 directions.

A second technique has been developed in order to overcome this major drawback. All pixels whose value was greater than a given fraction of the maximum pixel value for the study were selected to define the so called isodensity ROI. 
This technique has the advantage of being automatic but it requires an unbiased calibration of each tomographic slice to be fully optimal. Although one would expect it to be very sensitive to the statistical fluctuations associated with a single pixel value (namely the maximum) it provides stable results, depending on the true contrast and on the threshold used, as can be seen on table $V$. The number of pixels in the isodensity region is variable and take into account the statistical fluctuations in the image. In all cases the region formed was a closed one except when the contrast was 0.55 and the threshold was 0.5 . In that situation several pixels rrom the background, randomly distributed, were included in the region and the resulting ROI value was unstable. The optimal threshold to be used is then strongly dependant on the true contrast between the two regions. Using a threshold of 0.9 the $\mathrm{RC}$ was $0.72 \pm 0.02$ (mean $\pm \mathrm{SD} ; \mathrm{CR}=1.0$ ) and $0.77 \pm 0.02$ (mean $\pm \mathrm{SD} ; \mathrm{CR}=0.55$ ). This threshold is adequate at the two contrast levels but might be to low at lower contrast. The technique however could be used in conjunction with a mask filter that would isolate the BG in the PET image to prevent the selection of pixels not belonging to the $\mathrm{BG}$.

\section{Table IV}

$\mathrm{RC}$ values for single $\mathrm{ROI}$ and integrated $\mathrm{ROI}$ (single isotope experiments)

\begin{tabular}{|c|c|c|}
\hline BED POSITION & Single ROI & Integrated ROI \\
\hline 0 & 0.74 & 0.59 \\
\hline 1 & 0.71 & 0.58 \\
\hline 2 & 0.76 & 0.54 \\
\hline 3 & 0.80 & 0.57 \\
\hline 0 & 0.74 & 0.59 \\
\hline
\end{tabular}

Table V

$\mathrm{RC}$ values for isodensity $\mathrm{ROI}$ as a function of threshold and true contrast (single isotope experiments)

\begin{tabular}{|c|c|c|c|c|}
\cline { 2 - 5 } \multicolumn{1}{c|}{} & \multicolumn{4}{c|}{$\mathrm{CR}=1.0$} \\
\cline { 2 - 5 } \multicolumn{1}{c|}{} & \multicolumn{3}{c|}{ Threshold : 0.5} & \multicolumn{2}{c|}{ Threshold : 0.9} \\
\hline $\begin{array}{c}\text { Bed } \\
\text { position }\end{array}$ & \# pixels & $\begin{array}{c}\text { Isodensity } \\
\text { ROI }\end{array}$ & \# pixels & $\begin{array}{c}\text { Isodensity } \\
\text { ROI }\end{array}$ \\
\hline 0 & 294 & 0.55 & 61 & 0.73 \\
\hline 1 & 319 & 0.54 & 61 & 0.70 \\
\hline 2 & 345 & 0.54 & 38 & 0.70 \\
\hline 3 & 285 & 0.55 & 44 & 0.75 \\
\hline 0 & 307 & 0.55 & 59 & 0.73 \\
\hline & \multicolumn{3}{|c|}{$\mathrm{CR}=0.55(\mathrm{BG} / \mathrm{BK}=3.5)$} \\
\hline 0 & 633 & 0.43 & 40 & 0.77 \\
\hline 1 & 509 & 0.52 & 25 & 0.77 \\
\hline 2 & 1014 & 0.47 & 34 & 0.74 \\
\hline 3 & 428 & 0.55 & 39 & 0.78 \\
\hline 0 & 354 & 0.56 & 64 & 0.78 \\
\hline
\end{tabular}

\section{CONCLUSION}

We have built a model of the human basal ganglia and characterized its recovery coefficient under various experimental conditions on our tomograph. Our data illustrates the difficulty in trying to derive a single correction factor to be applied on a measured value of activity concentration in the BG. The RC is a function not only of the size and shape of the structure and the resolution of the counting instrument, but also on its contrast with surrounding tissues and to some extent on its axial position within the tomographic slice.

Accuracy and precision of our data have been extensively discussed. Precision, which is important when reproducibility is required for the correct interpretation of data, is affected by the contrast. The higher the contrast the more sensitive the value of RC is to mispositioning. 'This can be improved by using larger ROIs or by integrating measured values over several tomographic slices creating a 3-Dimensional ROI. However the decrease of variability is often accompanied by an increase of quantitation loss.

Total recovery, under any conditions, can only be achieved if the partial volume effect is reduced and the effect of scattered radiation diminished (through direct rejection or through the elaboration of an adequate correction scheme). We have shown, with data obtained from a dual isotope experiment, that a constant fraction from the background is added to the measured data in the BG. The consequences of such perturbations on the evaluation of physiological parameters should be further investigated.

\section{ACKNOWLEDGMENT}

The authors would like to thank J. S. Fowler and Y. Hodé for helpful discussions. They also would like to thank C. Koehler, R. Becker and E. Ritter for advices and assistance in the construction of the phantom, D. Warner and N. Pappas for their technical assistance and P. Luk (CTI) for the scatter correction algorithm.

\section{REFERENCES}

[1] E.J. Hoffman and M.E. Phelps, "Positron Emission Tomography: principles and Quantitation", in Positron Emission Tomography and Ausoradiography: Principles and Applications for the Brain and the Heart, New York: Raven Press, 1986, pp 237-286.

[2] H.N. Wagner Jr, "Quantitative Imaging of Neuroreceptors in the Living Human Brain,", Seminar in Nuclear Medicine, vol. XVI, pp. 51-62, January 1986.

[3] G. Sedvall, L. Farde, A. Persson, and F. Wiesel, "Imaging of Neurotransmitter Receptors in the Living Human Brain,", Arch Gen Psychiatry, vol 43, pp. 995-1005, Oct 1986.

[4] B. Mazière and M. Mazière, "Where have we got to with neuroreceptor mapping of the human brain,", Eur J Nucl Med, vol 16, pp.817-835, 1990.

[5] N.C. Andreasen, R. Carson, M. Diksic, A. Evans, L. Farde, A. Gjedde, A. Hakim, S. Lal, N. Nair, G. Sedvall, L. Tune and D. Wong, "Workshop on Schizophrenia, PET, and Dopamine D2 
Receptors in the Human Neostriatum,". Schizophrenia Bulletin, vol. 14(3), pp.471-484, 1988

[6] J.S. Fowler and A.P. Wolf, "Positron-Emitter Labelled compounds: Priorities and Problems." in Positron Emission Tomography and Auloradiography: Principles and Applications for the Brain and the Heart, New York: Raven Press, 1986. pp $391-450$.

[7] J. Delforge, A. Syrota, and B. Mazoyer, "Identifiability Analysis and Parameter Identification of an In Vivo LigandReceptor Model from Pet Data,", IEEE Trans on Biomedical Engineering, Vol. 37(7), pp. 653.661, July 1990.

[8] E.J. Hoffman, S.C. Huang, and M.E. Phelps, "Quantitation in Positron Emission Computed Tomography: 1. Effect of Object Size,", J. of Comput. Assist Tomog.. Vol 3(3), pp. 299-308, June 1979.

[9] J.C. Maziotta, M.E. Phelps, D. Plummer, and D.E. Kuhl, "Quantitation in Positron Emission Computed Tomography: 5. Physical - Anatomical Effects,", J. of Comp. Assist. Tomog., Vol 5(5), pp. 734-743, October 1981.

[10] R. M. Kessler, J.R. Ellis Jr, and M. Eden, "Analysis of Emission Tomographic Scan Data: Limitations Imposed by Resolution and Background,", J. of Comp. Assist. Tomog., Vol 8(3), pp. 514-522, June 1984.

[11] J. S. Fowler and A.P.Wolf, "New Directions in Positron Emission Tomography,". Annual Reports in Medicinal Chemistry, Vol 24, pp 277-286, 1989 (and ref therein).

(12) S.M. Blinkov and I.I. Glezer, The Human Brain in Figures and Tables. A quantitative handbook, Plenum Press 1968, pp. $166-170$.

[13] B. Bendriem, S.L. Dewey, and D.J. Schlyer, " Positioning Error Induced By Axial Sampling in Positron Emission Tomography," in Proc. of SPIE meeting on Science and Engineering of Medical Imaging, Paris, April 1989, Vol 1137, pp. 78-83.

[14] C. D. Arnett, A. P. Wolf, C.Y. Shiue, J. S. Fowler, R. R. MacGregor, D. R. Christman, and M. R. Smith, "Improved Delineation of Human Dopamine Receptors Using. [F-18]- N. Methylspiroperidol and PET.", J. of Nucl. Med., vol 27, pp 1878-1882, 1986.

[15] T. J. Spinks, T. Jones, M. C. Gilardi, and J.D. Heather, "Physical Performances of the Latest Generation of Commercial Positron Scanner,", IEEE Trans. on Nucl. Sci., vol NS-35, pp. 721-725, February 1988.

[16] B. E. Cooke and A.C. Evans," A Phantom to Assess Quantitative Recovery of Positron Tomographs,". J. of Comp. Assist. Tomog., Vol 7(5), pp. 876-880, October 1983.

[17] M. Bergstrom, L.Eriksson, C. Bohm, G.Blomqvist, and J. Litton," Correction For Scattered Radiation in a Ring Detecior Positron Camera by Integral Transformation of the Projection," , J. of Comp. Assist. Tomog., Vol 7(1), pp. 42 50, February 1983.

[18] E.J. Hoffman, S.C. Huang, D. Plummer, and M.E. Phelps, "Quantitation in Positron Emission Computed Tomography: 6. Effect of Non Uniform Resolution .," J. of Comput. Assist Tomog., Vol 6(5), pp. 987-999, October 1982.

\section{DISCLAIMER}

This report was prepared as an account of work sponsored by an agency of the United States Government. Neither the United States Government nor any agency thereof, nor any of their employees, makes any warranty, express or implied, or assumes any legal liability or responsibility for the accuracy, completeness, or usefulness of any information, apparatus, product, or process disclosed, or represents that its use would not infringe privately owned rights. Reference herein to any specific commercial product, process, or service by trade name, trademark, manufacturer, or otherwise does not necessarily constitute or imply its endorsement, recommendation, or favoring by the United States Government or any agency thereof. The views and opinions of authors expressed herein do not necessarily state or reflect those of the United States Government or any agency thereof. 

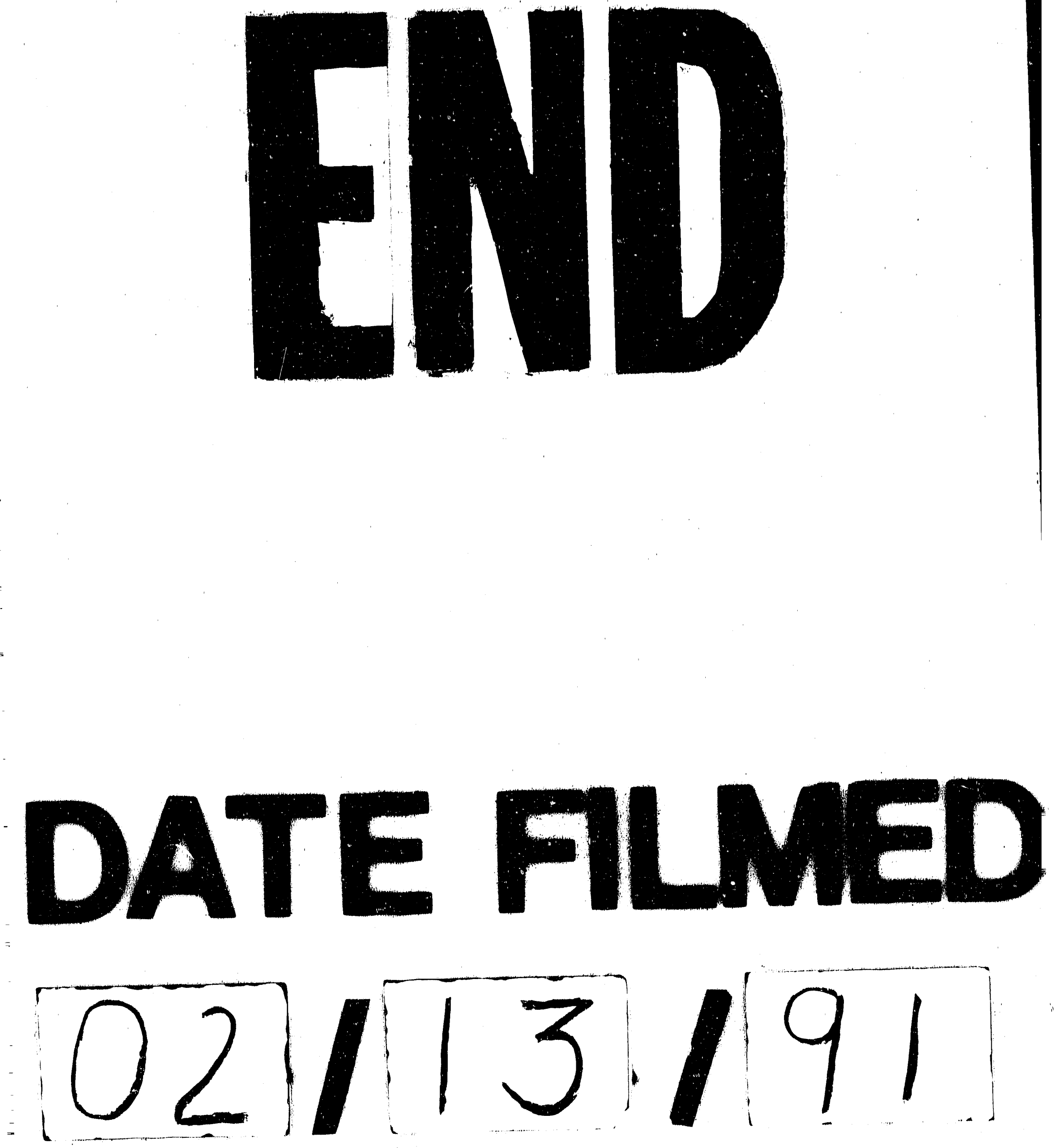
